P68 (continued)

young children throughout the state. Michigan State University Extension designed a lesson plan and cooking demonstration based on the needs of the families, and delivered the presentations in Spanish to 651 adults at 14 Michigan Migrant Head Start sites. The presentation included information about healthy fruit and vegetables, how to eat fewer calories, the importance of drinking water, how to maintain a healthy weight, low fat proteins and dairy, and the importance of physical activity.

Evaluation: As a result of the presentations, $85 \%$ of participants said they planned to eat more vegetables; $92 \%$ said they planned to eat more fruits; $93 \%$ said they would drink more water; and 80\% said they would do more physical activity.

Conclusions and Implications: The poster presentation will elaborate on the design, implementation, and evaluation of the program.

Funding: Supplemental Nutrition Assistance Program Education

\section{P69 WITHDRAWN}

\section{P70 Effectiveness of Train the Trainer Methodology for Delivery of a Preschool Nutrition and Physical Activity Curriculum}

Kaylee Karsky, BS, South Dakota State University; Kendra Kattelmann, PhD, RDN, LN, FAND, kendra. kattelmann@sdstate.edu, South Dakota State University, Box 2203, Wagner 443, Brookings, SD 57007; Howard Wey, PhD, South Dakota State University; Lacey McCormack, PhD, MPH, RD, LN; Mary Bowne, EdD; Suzanne Stluka, MS, RDN; Tara Shafrath, MS; Karlys Wells, MS, MEd; Jessica Meendering, PhD

Objective: To determine the structural fidelity of implementation (FOI) of a nutrition and physical activity (PA) curriculum by preschool teachers trained through trainthe-trainer.

Study Design, Setting, Participants: The intervention (iGrow Readers), a cluster-randomized field study in preschool centers, was based on children's books paired with nutrition and PA lessons. All teachers were trained by the author of iGrow Readers. The presentation of lessons was reviewed for FOI in randomly chosen teachers, resulting in review of 92 lessons (14 teachers). All FOI reviews were completed by one researcher.

Outcome Measures and Analysis: The structural FOI included 7 questions that queried for meeting the objective, introducing the lesson, previewing questions, allowing time to answer questions, analyzing material adequacy, using pictures, and referring to the guide. Questions were analyzed individually using generalized linear mixed models with logit link (observations nested within teacher) to estimate an intra-class correlation coefficient (ICC). Four of the questions were combined as a summary score (0-4) and used to evaluate the associations of lesson type (PA vs. nutrition) and class size using a mixed-effects model.

Results: Eighty-four percent of lessons had moderatelyhigh to high FOI (summary score3). The ICC for individual FOI questions varied from near zero to $82 \%$. The delivery of lessons was independent of lesson type (nutrition or PA). The ICC for the summary FOI score was 0.3 (95\% CI: $0.11,0.60)$, indicating heterogeneity in delivery.

Conclusions and Implications: There were variations in delivery of curriculum by preschool teachers trained through train-the-trainer, but overall FOI was high. Nonetheless, more rigorous training for preschool teachers may improve implementation of nutrition and PA curriculum.

Funding: USDA

\section{P71 Effects of the Slow Down Program: A Mindfulness-Based Stress Management and Nutrition Program for Mothers with Young Children}

Lauren Kennedy,PhD, kennedy0@vt.edu, Virginia Tech, 327 Wallace Hall, 295 West Campus Drive, Blacksburg, VA 24060; Sarah Misyak, PhD, Virginia Tech; Elena Serrano, $P h D$

Objective: To determine the impact of a mindfulnessbased stress management and nutrition program, the Slow Down Program (SDP), on mothers' eating behavior, stress, and child feeding practices.

Theory, Prior Research, Rationale: The SDP is based upon the neuropsychological model of obesity that addresses the relationship between psychological stress and eating. Fishbein's Integrated Model for behavior change was used to guide program planning.

Description: The SDP was a four-week program designed to help mothers develop a toolbox of mindfulness-based skills to manage stress, practice mindful eating behaviors, and learn about healthy eating for themselves and their families. The SDP uses skills-based guided meditation, goal setting, and facilitated dialogue.

Evaluation: Measures collected at baseline and postintervention included: dietary patterns, child feeding practices, eating behavior, perceived stress, and self-efficacy toward healthy eating, stress reduction, and mindfulness. Mothers also completed weekly acceptability forms and participated in a focus group discussion at the conclusion of the program and individual interviews four to six weeks after the program.

Conclusions and Implications: Participants reported that the SDP facilitated behavior change and self-efficacy in mothers for practicing mindfulness, managing stress effectively, and eating healthfully. Additionally, the benefit of having safe, nonjudgmental opportunities to share personal stories and challenges assisted with

Continued on page S51 
P71 (continued)

stress reduction. Nutrition education programs for adults should incorporate stress reduction and mindfulness to support whole health in addition to nutrition. Funding: None

\section{P72 Measuring Plate Waste with Family-Style Meals in Head Start: A Pilot Study \\ Cody Parris, BS, East Carolina University; Kristey Coulter, BS, Pitt County Health Department; Virginia C. Stage,PhD, LDN, RDN, carrawaystagev@ecu. edu, East Carolina University, Health Science Building 4310J, East Carolina University, Greenville, NC 27834}

Objective: Develop and pilot-test a standardized protocol for plate waste of family-style meals in Head Start

Study Design, Setting, Participants: A standardized protocol was developed after five observations. Four researchers pilot-tested the protocol by collecting plate waste and observational data in two Head Start classrooms in eastern NC during four seperate lunches. Approximately 18 low-income 3-5-year-old children were in each classroom.

Outcome Measures and Analysis: Plate waste was collected for 8 observations on 4 different days. Milk, entrée, vegetable, and fruit were measured. Before lunch, food was measured in large serving bowls. Post mealtime measurements merged all waste for each food group into its original bowl. Descriptive statistics were used to analyze waste percentage. Researchers identified challenges for collecting plate waste with family-style meals using observational notes.

Results: Overall percent wasted was approximately $26 \%$. Fruit was the most wasted (29.31\%), followed by vegetables (27.39\%) and milk (27.13\%). Entrees had the lowest waste (19.54\%). The most wasted individual item was salad (60.69\%); the least Beefaroni (7\%). Challenges to measuring family-style plate waste included measuring spilled fluids, separating foods combined during the meal, measuring inedible portions, and measurement of waste for individual children.

Conclusions and Implications: Standardized methods for measuring plate-waste are needed to accurately assess dietary intake in settings that use family-style dining. Further refinement of the researcher-developed plate waste protocol is needed based on challenges observed during pilot-testing. More research is needed to develop standards for a wider variety of meals, and to determine feasible methods for measuring plate waste for individual children.

Funding: None

\section{P73 A Descriptive Assessment of Weekend Food Programs and the Impact on Household Food Security}

Lesli Biediger-Friedman, PhD, MPH, RD, LB47@txstate.edu, Texas State University, 601 University Drive, San Marcos,
TX 78666; Xiunely R. Cadenas, BS, Texas State University; Tiffany Rodriguez

Objective: A growing approach to address weekend food insecurity includes weekend backpack food programs. Little is known about the impact these programs have on a child's household food. The purpose of the study was to describe the impact of two weekend food programs on household nutrition and food security.

Study Design, Setting, Participants: The study examined programs serving elementary school-aged students enrolled in one of two weekend food programs in a suburban county of south central Texas. In depth interviews were conducted with a purposeful sample of 8 Englishspeaking and 8 Spanish-speaking parents of student participants. Interview questions explored participation with other food resources, the impact of the food provided to household food security and nutrition, and child acceptance of the food provided.

Outcome Measures and Analysis: Interviews were immediately transcribed and analyzed using a threecoder emergent theme coding structure. Meta themes included reduction of food portions with reported characteristics of food insecurity, acceptance of the food by parents and children, and a described improvement to nutritional variance of the household foods. Attitudes toward the weekend food programs were generally positive, however, some responses were critical of the nutrition quality of highly processed foods. Though the food program provided food only for the school student, all participants discussed household sharing of the food provisions. Participants wanted larger portions of food, and more protein, fruits, and vegetables.

Conclusion and Implications: Weekend food programs are a promising approach to improve food security of families and provide nutritious foods. However, complex household dynamics revealed that many participants were still food insecure.

Funding: None

\section{P74 Analysis of Sugar-Sweetened Beverage Purchases by Supplemental Nutrition Assistance Program Participants}

Andres Romualdo, MA, andresp.romualdo@gmail.com, IMPAQ International, LLC, 10420 Little Patuxent Parkway, Suite 300, Columbia, MD 21044

Objective: This study examines the differences of item level sugar-sweetened beverage (SSB) purchases between two groups: Supplemental Nutrition Assistance Program (SNAP) households and non-SNAP households.

Study Design, Setting, Participants, Intervention: An exploratory study was conducted to examine the differences in SSB purchases between SNAP recipients and non-SNAP participants who have loyalty cards with a major, national food retailer. 